\title{
Human Facial Expression Recognition using Gabor Filter Bank with Minimum Number of Feature Vectors
}

\author{
Priya Sisodia \\ M.Tech (Computer Science \\ Engg.) \\ Ajay Kumar Garg \\ Engineering College, \\ Ghaziabad, India.
}

\author{
Akhilesh Verma \\ Asst. Prof (Computer \\ Science Engg.) \\ Ajay Kumar Garg \\ Engineering College, \\ Ghaziabad, India.
}

\author{
Sachin Kansal \\ $\mathrm{PhD}$ (Mechanical Department) \\ IIT Delhi, \\ Delhi, India
}

\begin{abstract}
The Human Facial Expression Recognition is used in many fields such as mood detection and Human Computer Interaction (HCI). Gabor Filters are used to extract features. Gabor has the useful property of robustness against slight object rotation, distortion and variation in illumination. In the present work the effort has been made to provide the modules of for Human facial expression recognition by reducing the number of parameters use to represent Gabor feature the space complexity can reduce. SVM classifier has multi-classes. SVM classifies the expression by comparing it with the trained data.
\end{abstract}

\section{Keywords}

Image Acquisition, Preprocessing, Feature Extraction, Classification.

\section{INTRODUCTION}

Human facial expression recognition is defined as a change that happens in response to human internal emotional states. At the time of human communication, facial expression played a major role. Major component of human communication is facial expression which constitutes around $55 \%$ of the total communicated message. The basic facial expression that recognized by psychologists are: neutral, happiness, sadness, anger, fear, surprise and disgust.

Applications of Human Facial Expression recognition system are psychological Studies, social interaction, Human Computer Interaction (HCI) (Ekman et al., 1997).

There are many methods have been proposed for human facial expression recognition from static images (image database) to image sequence (video) (B. Fasel et al., 2003).

Human facial expression analysis is a computer system that automatically analyzes and recognizes facial features and classifies those (Fernando De La Torre et al., 2011).

Following steps involve in Human Facial expression recognition system are:-

1. Preprocessing: this step comprises of operations like image scaling, image brightness and contrast adjustment and other image enhancement operation.

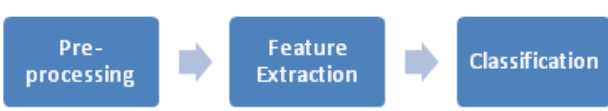

Figure 1: General Structure of Human Facial expression Analysis System

The Human Facial Expression system uses an image database to train and test the performance of the classifier.

2. Feature Extraction: the key parameters that efficiently represent the particular facial expression need to be extracted from the images. These parameters are used to discriminate between expressions.

3. Classification: Feature vector of test image is compared with feature vector of trained database and classify them accordingly (Shishir Bashyal et al., 2008).

Techniques under Feature Extraction phase and Classification Phase are as follows (Ying-Li Tian et al., 2005).

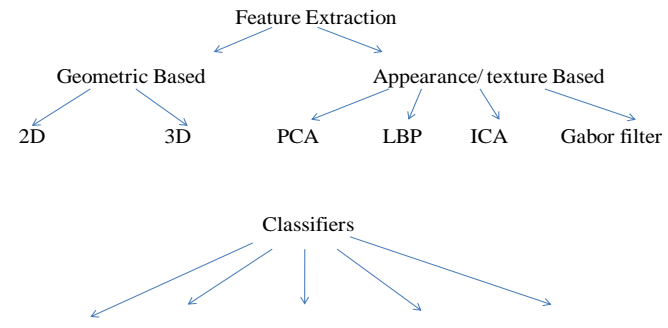

Figure 2: Techniques for Feature Extraction and Classifier

Appearance based approach outperforms Geometric based approach (Zhengyou Zhang et al., 1998).

There are numbers of technique for facial expression analysis but no single method is efficient with respect to memory and time complexity. 
The existing facial expression system may misclassify certain expressions. This is due to facial expressions in training database may be different from spontaneous expressions (Fei Long et al., 2012).

\section{RELATED WORK}

Many techniques have been used for Human Facial Expression Recognition from 1978 onwards.

Andrew J. Calder et al. (2001) used principal component analysis (PCA) and linear discriminant analysis (LDA). Facial images were submitted to a PCA. PCA converted them into eigenfaces then the output of PCA was submitted to a series of LDA. 4 sets of PCA data showed good categorization rate. Performance was about $86 \%$.

Ira Cohen et al. (2003) used tracking point and Hidden Markov Model (HHM) to develop a system that interacts with the user. Author first time used HHM based classification which combines the temporal information to perform the classification. Explored both person-dependent and personindependent recognition of expressions and compared the different methods. The performance of the system was completely depends on the database.

Hong-Bo Deng et al. (2003) used local Gabor filter bank and PCA plus LDA. A novel local Gabor filter bank with part of orientation and frequency parameters was proposed. Two stage feature compression method (PCA plus LDA) used to select and compress the Gabor feature. The minimum distance classifier was adopted to recognize facial expression.

Spiros V. Loannou et al. (2005) used the Neurofuzzy Network to develop robust system which must be person-independent. Neurofuzzy Network System has learning/ adaptation and inference rules for knowledge refinement. The performance of the system was about $78 \%$.

Jun Wang, Lijun Yin (2007) used topographic context (TC) and linear discriminant analysis (LDA). This system works as robust and person-independent. A Topographic analysis treats the image as a 3D surface and labels each pixel by its terrain features. Topographic context describes the distribution of topographic labels in a region of interest of a face (split face into a number of expressive regions). These regions are combined with terric map. LDA was used as a classifier and performance of the system was about $82.61 \%$.

Praseeda Lekshmi V. et al. (2008) used Principal component analysis for feature extraction and weight vectors for classification. Skin color detection was used to detect skin region. At the time of pre-processing 14 marking points were mentioned on feature face. In PCA, facial images were projected into a feature space or Eigen space. They have combined geometric and Eigen face at classification phase. The performance of the system was about $88 \%$.

Shishir Bashyal et al. (2008) used Gabor filter and learning vector quantization to develop a facial expression recognition system. Gabor filter was used to extract the feature from JAFFE database images. This paper proposed that learning vector quantization (LVQ) performs better in recognition of fear expressions than multilayer perceptron (MLP). Performance of recognition rate was about $90.22 \%$

Caifeng Shan et al. (2009) used Linear Binary Pattern for feature extraction and Support Vector Machine (SVM) for classification. Developed a system that works against illumination changes and take less time as others existing system. Linear Binary Pattern (LBP) tolerant illumination and takes less time in computation. The performance of the system was about $89 \%$ for sadness. Some expressions were not classified correctly.

Luiz S. OLiveira et al. (2011) used 2 Dimension Principal Component Analysis for feature extraction and K-Nearest Neighbor for classification. They tried to solve some problems of the PCA which effect recognition problem. As PCA works on vector image while 2DPCA works on the whole image. But in 2DPCA coefficient are more so, we need to select feature for classification. The performance of the system was about $91 \%$.

Dahmane M. et al. (2011) developed a system to implement the general expression model (even when a person poses at different time). They used Histrograms of oriented gradients (HOG) and Support Vector Machine (SVM). The baseline method was used. HOG is used to extract the appearance feature by gradient magnitudes for a set of orientations. The performance of the system was about $70 \%$.

Le Hoang Thai et al. (2011) used Canny, Principal Component Anlysis and Artificial Neural Network for classification of facial expression. Canny was applied for preprocessing phase to locate the region of detection. After that PCA was applied to extract the facial features. At last Artificial Neural Network applied to classify the facial expression. The performance of the system was about $85.7 \%$.

Wenfei Gu. et al. (2012) used Gabor filter for feature extraction and Classifier synthesis. The system supports person- independent expression recognition and variation in illumination. Gabor filters have the useful property of robustness against slight object rotation, distortion and variation in illumination. The performance of the system was $91.5 \%$.

The techniques used by researchers previously had certain problems. Some of them only give good result for persondependent test. If the test input was not from the trained database then the results were different.

In many techniques miss-classification of facial expressions was common, due to low intensity of expressions.

Results were good for particular expression only like- only neutral, only sad, only happy etc.

The overall summary is given in Table I. This table summarized all the techniques used by authors.

\section{METHODOLOGY}

Figure 7 shows the flow diagram of method for Human Facial Expression recognition. This method is divided in three parts: preprocessing phase, feature extraction phase, classification phase.

\subsection{Image Acquisition:}

In image acquisition phase, there are several issues such as: the properties and the number of video cameras and digitizer, the size of face space in comparison to total image dimensions, and the low lighting conditions. These factors affect facial expression recognition process. Images captured in low lighting condition or at low resolution can provide less information about facial features. If the face image size is small as compared to total image size then less information is available about the image. 
Table I: summary of techniques used for Facial Expression Recognition

\begin{tabular}{|c|c|c|c|c|c|c|}
\hline Year & Author & $\begin{array}{c}\text { Problem } \\
\text { Formulation }\end{array}$ & Solution & Features Extraction & Classification & $\begin{array}{c}\text { Performance } \\
\text { Rate }(\%)\end{array}$ \\
\hline 2001 & $\begin{array}{c}\text { Andrew J Calder et } \\
\text { al. }\end{array}$ & $\begin{array}{l}\text { Can PCA code facial } \\
\text { expression in a form } \\
\text { that can support their } \\
\text { recognition? }\end{array}$ & $\begin{array}{l}\text { Yes and } 4 \text { set PCA data } \\
\text { showed good } \\
\text { categorization rate. }\end{array}$ & PCA & LDA & 86 \\
\hline 2003 & Ira Cohen et al. & $\begin{array}{l}\text { To develop a system } \\
\text { that interact with the } \\
\text { user }\end{array}$ & $\begin{array}{l}\text { First time use Hidden } \\
\text { Markov Model (HMM) } \\
\text { classifier (combine } \\
\text { temporal info.) }\end{array}$ & Tracking feature point & HMM & 85 \\
\hline 2005 & $\begin{array}{l}\text { Spiros V. Loannou } \\
\text { et al. }\end{array}$ & $\begin{array}{l}\text { To develop robust } \\
\text { system and facial } \\
\text { action person } \\
\text { variation }\end{array}$ & $\begin{array}{c}\text { NeuroFuzzy N/W } \\
\text { System has } \\
\text { learning/Adaptation and } \\
\text { Inference rules for } \\
\text { knowledge refinement }\end{array}$ & Neurofuzzy Network & Rules based & 78 \\
\hline 2007 & Jun Wang et al. & Robust System & $\begin{array}{l}\text { Topographic context } \\
\text { describes the } \\
\text { distribution of } \\
\text { topographic label in a } \\
\text { region of interest of a } \\
\text { face ( split face into no. } \\
\text { of expressive region) }\end{array}$ & $\begin{array}{l}\text { Topographic Context } \\
\text { (TC) }\end{array}$ & LDA & 82.61 \\
\hline 2008 & $\begin{array}{l}\text { Praseeda Lekshmi } \\
\text { et al. }\end{array}$ & $\begin{array}{l}\text { Shape+ location and } \\
\text { whole image work. }\end{array}$ & $\begin{array}{l}\text { Combine geometric and } \\
\text { Eigen face at } \\
\text { classification step }\end{array}$ & PCA & Weight Vector & 88 \\
\hline 2008 & $\begin{array}{l}\text { Shishir Bashyal et } \\
\text { al. }\end{array}$ & $\begin{array}{c}\text { Improve } \\
\text { performance of } \\
\text { recognition phase. }\end{array}$ & $\begin{array}{l}\text { Proposed that learning } \\
\text { vector quantization } \\
\text { (LVQ) performs better } \\
\text { in recognition of fear } \\
\text { expressions than } \\
\text { multilayer perceptron } \\
\text { (MLP) }\end{array}$ & Gabor Wavelet & LVQ & 90.22 \\
\hline 2009 & Caifeng Shan et al. & $\begin{array}{l}\text { To build a system } \\
\text { that works against } \\
\text { illumination, } \\
\text { changes and take } \\
\text { less time as the other } \\
\text { existing system }\end{array}$ & $\begin{array}{l}\text { Use Linear Binary } \\
\text { Pattern (LBP) that } \\
\text { tolerant illumination and } \\
\text { taken less time in } \\
\text { computation. }\end{array}$ & LBP & SVM & 89 \\
\hline 2011 & $\begin{array}{l}\text { Luiz S. OLiveira et } \\
\text { al. }\end{array}$ & $\begin{array}{l}\text { To solve some of } \\
\text { PCA's } \\
\text { Computational } \\
\text { problems (which } \\
\text { effect recognition } \\
\text { problem) }\end{array}$ & $\begin{array}{l}\text { Modify the PCA by } \\
\text { working on the whole } \\
\text { image. }\end{array}$ & 2DPCA & $\mathrm{K}-\mathrm{NN}$ & 91 \\
\hline 2011 & Dahmane M. et al. & $\begin{array}{c}\text { System to implement } \\
\text { the general } \\
\text { expression model ( } \\
\text { even when a person } \\
\text { poses at different } \\
\text { time) }\end{array}$ & $\begin{array}{l}\text { The baseline method } \\
\text { was used. HOG is used } \\
\text { to extract the } \\
\text { appearance feature by } \\
\text { gradient magnitudes for } \\
\text { a set of orientations }\end{array}$ & $\begin{array}{c}\text { Histogram of Oriented } \\
\text { Gradient }\end{array}$ & SVM & 70 \\
\hline 2012 & Wenfei Gu. et al. & $\begin{array}{l}\text { Person- independent } \\
\text { expression } \\
\text { recognition }\end{array}$ & $\begin{array}{l}\text { Gabor filters have the } \\
\text { useful property of } \\
\text { robustness against slight } \\
\text { rotated object, distortion } \\
\text { and variation in } \\
\text { illumination. }\end{array}$ & Gabor Filter & Classifier synthesis & 91 \\
\hline
\end{tabular}




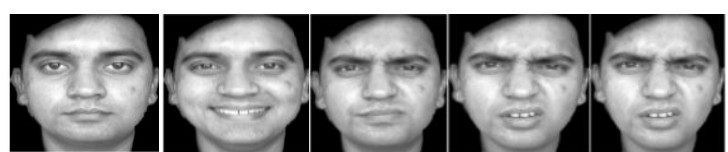

Figure 3: Sample of expression images.

\subsection{Preprocessing Phase:}

In Preprocessing Phase, the input image is processed and converted into the input which is able to feature extraction phase.

- Convert RBG image into a grayscale image.

- Detect faces from the input image.

- $\quad$ Resize the image before input it into the next phase.

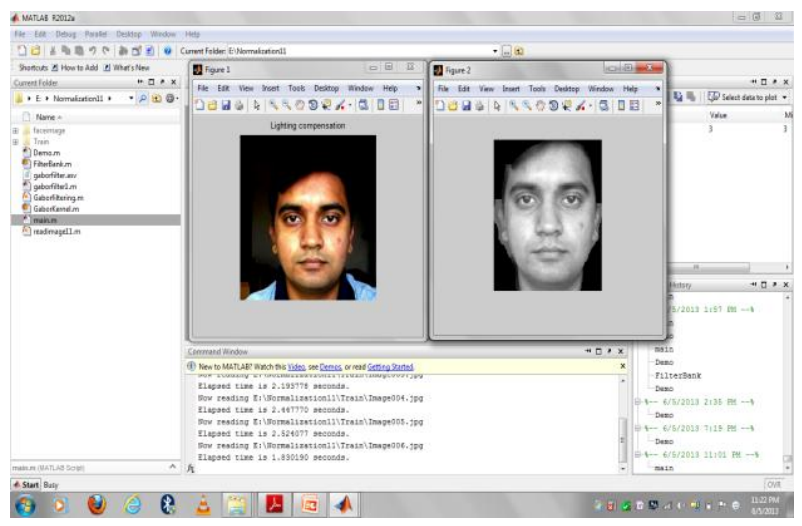

Figure 4: Example of Face detection on sample database

\subsection{Feature Extraction Phase:}

Features of images define the properties of an image on the basis of which the image is identified in the next phase.

Gabor Filters are used to select features that are necessary to represent expressions. There are 5 scales and 8 orientations of Gabor filter in general that can represent feature for each image. The Gabor Wavelet is defined as

$$
\psi(z)=\frac{p_{u, r}^{2}}{\sigma^{2}} \exp \left(-\frac{p_{u, r}^{2} Z^{2}}{2 \sigma^{2}}\right)\left[\exp \left(i p_{u, r} Z\right)-\exp \left(-\frac{\sigma^{2}}{2}\right)\right]
$$

Where $\mathrm{z}=(\mathrm{x}, \mathrm{y})$, and $\mathrm{u}$ and $\mathrm{r}$ define the orientation and scale of the Gabor wavelet.

$P_{\mathrm{u}, \mathrm{r}}=\mathrm{P}_{\mathrm{r}} \mathrm{e}^{\mathrm{i} \phi \mathrm{r}}$, where $\mathrm{P}_{\mathrm{r}}=\mathrm{P}_{\max } / f^{\mathrm{r}}$ and $\phi_{\mathrm{u}}=\Pi \mathrm{u} / 8 . \mathrm{P}_{\max }$ is the max frequency, and $f$ is the spacing factor.

The Gabor filter produces two parts: the real part and the imaginary part. Real and imaginary parts are transformed into two kinds of Gabor feature: the magnitude and phase. There are 40 Gabor feature for each image. Gabor wavelet features computation is expensive, due to convolution of facial images with a set of Gabor wavelet. It has high redundancy in Gabor wavelet feature. We can select more significant Gabor feature to represent the expression of each image that reduce space complexity.

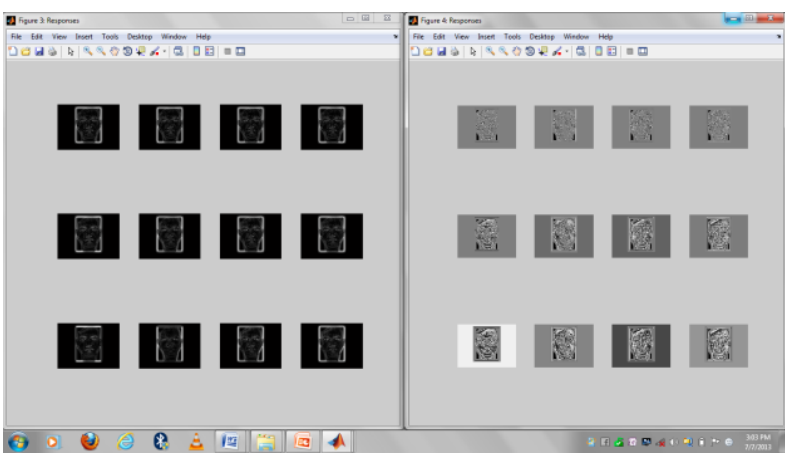

Figure 5: Example of Gabor wavelet representation of a face image shown in Fig. (a) The magnitude, (b) the real part of the resulting images.

\section{Classification Phase:}

Multiclass SVM is used to classify all different classes of expressions like happy, sad, surprise, fear, disgust, Anger, neutral. Here 6 classes for each expression are used for classification.

SVM convert Gabor Feature into vector form. When a test image is input, firstly Gabor is created for that image and converts it into a vector. In SVM the data is partitioned into the two parts that one is called Training set and other is known as the testing set and each having the instances of the attributes. Each instances having one target means class labels and several attribute. The goal of the SVM is that to produce the model which predicts the target value of instances in the testing set which are given by only attributes value. It is based on the supervised learning methods. Support vector machine has a unique property that it creates the hyperplane or a set of hyperplane which has taken into the consideration for the classification. It defines the functional margin means a good separation is achieved by the hyperplane that has the largest distance to the nearest data points of any class. Greater the margin it is easy to find better accuracy and minimal errors has found.

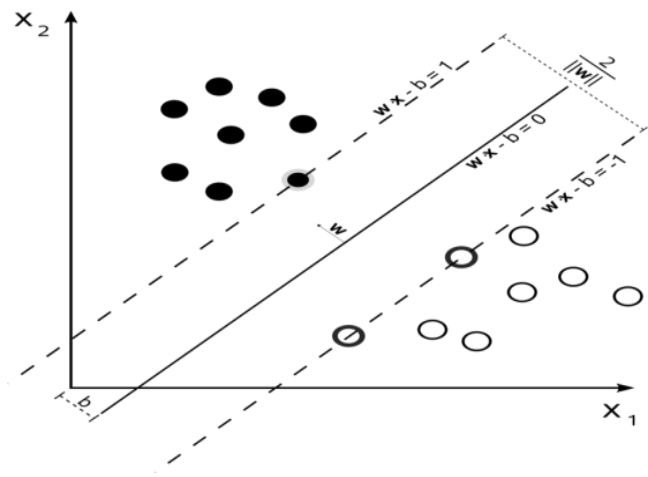

Figure 6. Hyperplane that discriminates between two classes. 


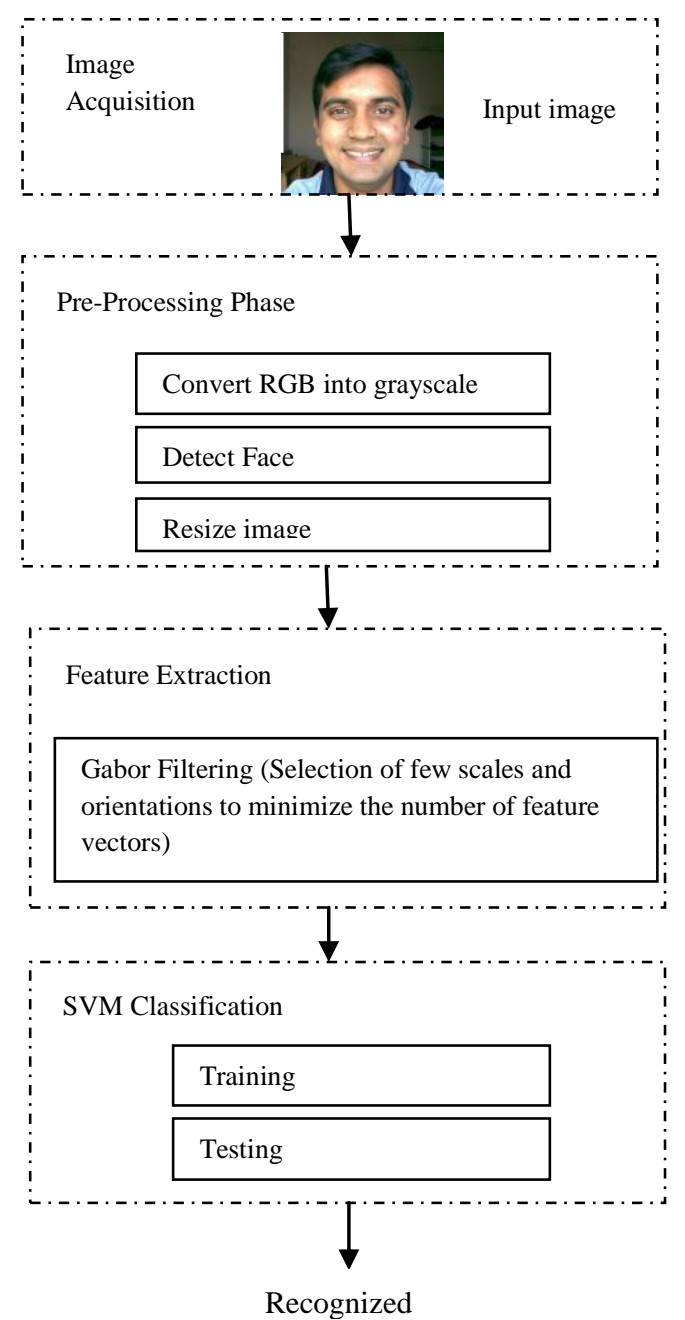

Figure 7: Flow Diagram of overall process of Human Facial Expression Recognition.

\section{CONCLUSION}

In this paper we have provided modules for Human Facial expression recognition using an appearance based algorithm. Gabor Filter outperformed the other existing techniques. It is capable to reveal both transient and intransient facial features. It removes variability in images that occurs due to changes in lighting and other noises. Selection of best Gabor Feature will reduce the space complexity of the system. The output of Gabor will be input into SVM classifier. SVM compares the test data with trained data and classify the expression accordingly.

\section{ACKNOWLEDGMENTS}

Our thanks go to the experts who have contributed towards development of this paper. To our project guide Mr. Akhilesh Verma for his valuable comments and sharing his knowledge. A special thank you goes Ajay Kumar Garg Engg College (Ghaziabad) for providing me good research environment.

\section{REFERENCES}

[1] Ekman, P., \& Rosenberg, E. L.," what the face reveals: Basic and applied studies of spontaneous expression using the Facial Action Coding System (FACS)", New York: Oxford University Press, 1997.

[2] B. Fasel, J. Luettin, "Automatic facial expression analysis: a survey”, Pattern Recognition, Vol. 36, pp 259-275, 2003.

[3] Fernando De La Torre \& Jeffrey F. Cohn, 'Facial Expression Analysis', Springer, VAH, pp 377-409, 2011.

[4]Shishir Bashyal, Ganesh K. Venayagamoorthy, "Recognition of Facial Expression using Gabor wavelets and learning vector quantization", Elsevier, EAAI 21, pp 1056-1064, 2009

[5] Ying-Li Tian, Takeo Kanade, and Jeffrey F. Cohn, "Facial expression Analysis", Springer Link, 2005.

[6] Zhengyou Zhang et al., "Comparison between Geometrybased and Gabor Wavelets-based facial expressions recognition using Multi-layer perception", IEEE conf., 1998.

[7] Fei Long et al., "Learning Spatiotemporal feature by using Independent component analysis with application to facial expression recognition", Elsevier Trans., Neurocomputing, 126-132, 2012.

[8] Andrew J. Calder, et al., "A Principal Component analysis of facial expressions", Elsevier Science Ltd Trans., 2001.

[9] Ceifeng Shan, et al., "Facial expression recognition based on Local Binary Patterns: A comprehensive Study", Elsevier Science Ltd Trans., 2009.

[10] Ira Cohen, et al., "Facial expression recognition from video sequences: Temporal and Static modeling", Elsevier Inc. Trans., 2003

[11] Jun Wang, Lijun Yin, "Static topographic modeling for facial expression recognition and analysis", Elsevier Science Ltd. Trans., 2007.

[12] M. Dahmane and J. Meunier, "Emotion recognition using dynamic grid based HOG feature", IEEE In. conf.,2011.

[13] Praseeda Lekshmi V., et al., "Analysis of Facial Expressions from Video Images using PCA", IEEE Conf., 2008.

[14] S. Luiz OLiveira, et al., "2D Principal Component Analysis for face and facial expression recognition", IEEE Trans., 2011

[15] Spiros V. Loannou, et al., "Emotion recognition through facial expression analysis based on a Neurofuzzy network”, Elsevier Science Ltd. Trans., 2005.

[16] Wenfei Gu, et al., "Facial expression recognition using radial encoding of local Gabor Feature and Classifier Synthesis", Elsevier Ltd Trans., 2012.

[17] Shishir Bashyal et al., "Recognition of facial expression using Gabor wavelet and learning vector quantization" ,Elsevier Trans. ,Engineering Application of Artificial Intelligence 21, 1056-1064, 2008.

[18] http://www.kasrl.org/jaffe_info.html.

[19] Wei-lun Chao, "Gabor wavelet transform and its application", R98942073.

[20] Ligang Zhang, "Facial Expression Recognition Using Facial Movement Features", IEEE Trans. , Affective Computing, Vol. 2, issue 4, 2011. 